\title{
Existence of Equilibrium in Common Agency Games with Adverse Selection*
}

\author{
Guilherme Carmona ${ }^{\dagger}$ \\ Universidade Nova de Lisboa
}

\author{
José Fajardo \\ IBMEC Business School
}

September 15, 2008

\begin{abstract}
We establish the existence of subgame perfect equilibria in general menu games, known to be sufficient to analyze common agency problems. Our main result states that every menu game satisfying enough continuity properties has a subgame perfect equilibrium. Despite the continuity assumptions that we make, discontinuities naturally arise due to the absence, in general, of continuous optimal choices for the agent. Our approach, then, is based on (and generalizes) the existence theorem of Simon and Zame (1990) designed for discontinuous games.
\end{abstract}

Keywords: Common Agency, Menu Games, subgame perfect equilibrium.

${ }^{*}$ We wish to thank Andrea Attar, Mehmet Barlo, Steffen Hoernig, Gwenäel Piaser, Luís Vasconcelos, an Associate Editor and three anonymous referees for very helpful comments. We thank also John Huffstot for editorial assistance. Any remaining error is, of course, our own.

${ }^{\dagger}$ Address: Universidade Nova de Lisboa, Faculdade de Economia, Campus de Campolide, 1099032 Lisboa, Portugal; Phone: (351) 21380 1671; Fax: (351) 21387 0933; email: gcarmona@fe.unl.pt.

${ }^{\ddagger}$ Address: IBMEC Business School, Av. Presidente Wilson 118, 20030 020, Rio de Janeiro, Brazil; Phone: (55) 214503 4162; Fax: (55) 214503 4168; email: pepe@ibmecrj.br. 


\section{Introduction}

In many important examples in multi-contracting mechanism design, several principals (attempt to) contract with a common agent to influence her choice. Such a common agency model has been the focus of much of the recent research in incentive theory. ${ }^{1}$

In the common agency model, principals offer a menu of contracts to the agent, who chooses one contract from those being offered. Although one could imagine more general communication channels between the principals and the agent, Martimort and Stole (2002), Page and Monteiro (2003), Peters (2001) and Peters (2003) have shown that such a procedure of offering menus of contracts is enough to characterize the set of equilibrium allocations. In fact, as Martimort (2006) points out, "what matters per se is not the kind of communication that a principal uses with his agent but the set of options that this principal makes available to the agent." This result, known as the delegation principle, implies that the common agency problem can be analyzed through a menu game.

However, in order for the delegation principle to be meaningful, an equilibrium must exist. In this paper, we present a solution to this problem by establishing a general existence theorem for menu games.

A menu game is defined as follows. ${ }^{2}$ First, the agent's type is drawn from a commonly known distribution. Then, the principals simultaneously choose a menu of contracts (defined as a closed subset of a compact contract space) without observing the agent's type. Finally, the agent chooses one contract (or one contract of each principal), knowing her type and the menus offered by the principals.

The subgame perfect equilibria of a menu game can be easily described by noting that a strategy for the agent induces a normal-form game between the principals. ${ }^{3}$ In

\footnotetext{
${ }^{1}$ See Martimort (2006) for a survey.

${ }^{2}$ Our formalization is based on Page and Monteiro (2003).

${ }^{3}$ Although researchers in this field typically focus on perfect Bayesian equilibrium, we note that the set of subgame perfect equilibria of a menu game coincides with the set of its perfect Bayesian equilibria.
} 
fact, this is a game where each principal has the set of all possible menus as his own pure strategy set and his payoff is determined by the choice of all principals together with the agent's strategy. Thus, a subgame perfect equilibrium consists of an optimal strategy for the agent and a Nash equilibrium for the normal-form game induced by such strategy.

The problem of existence of a subgame perfect equilibrium would then be trivial if there were a continuous (in the principals' actions) optimal strategy for the agent. Indeed, the normal-form game induced by such strategy would be continuous and standard existence theorems would apply. The difficulty with the existence of equilibrium is that, in general, no optimal strategy for the agent is continuous even if the agent has a continuous utility function, a compact action space and a continuous choice correspondence.

Nevertheless, in a sufficiently continuous menu game (e.g., a menu game with continuous payoff functions for the principals and the agent, as well as with compact choice sets and a continuous choice correspondence for the agent) discontinuities can only arise as a result of a discontinuous strategy for the agent. However, such discontinuities create no problem for the existence of equilibrium. Indeed, it follows from the above description of a subgame perfect equilibrium that we can regard the family of normal-form games induced by the agent's strategies as a game with an endogenous sharing rule as in Simon and Zame (1990) and, therefore, use their existence theorem to establish the existence of subgame perfect equilibria in menu games. In fact, a vector of menus defines a subset of payoffs for the principals, each of which corresponds to a particular strategy of the agent. This clearly defines a correspondence from principals' strategies into payoffs as required for a game with an endogenous sharing rule.

In order to use Simon and Zame's theorem in our setting, we need to generalize it to allow the payoff correspondence to depend, in a measurable way, on the agent's type. Using an approach similar to that of Simon and Zame (1990), we show that any such generalized game with endogenous sharing rules has a solution. This extension is non trivial because the agent's type space is not assumed to be compact, but merely 
complete and separable and the payoff correspondence is not assumed to be jointly upper hemi-continuous, but only upper hemi-continuous in the principals' choices and jointly measurable.

Once the above generalization is accomplished, we can easily obtain a subgame perfect equilibrium from a solution. In fact, the Borel implicit function lemma of Furukawa (1972) shows that when the payoff correspondence is the composition of players' payoff functions with some correspondence (interpreted as the optimal choice correspondence of players whose behavior is not explicitly modeled), then every measurable selection from the payoff correspondence can be obtained as the composition of players' payoff function and a measurable selection of this other correspondence. Combining our generalization with this result, we show that every menu game satisfying enough continuity properties has a subgame perfect equilibrium.

Independently, Monteiro and Page (2008b) have shown that the common agency game can be reduced to a game with an endogenous sharing rule, to which the existence theorem of Simon and Zame (1990) can be applied. They then show that the agent's equilibrium strategy can always be chosen to have a support no larger than then the number of principals plus one and, when the agent's type space is nonatomic, that it can be taken to be a pure strategy.

The existence of equilibrium in menu games has also been addressed by Page and Monteiro (2003) and Monteiro and Page (2008a). The main difference between our approach and the approach taken in these papers arises because they fix exogenously a tie-breaking rule for the agent (to be used when she is indifferent between several contracts) and then they establish simultaneously the existence of an optimal strategy for the agent satisfying the tie-breaking rule and equilibrium strategies for the principals. In contrast, we proceed by determining simultaneously the equilibrium strategies for the principals and for the agent without fixing exogenously any tie-breaking rule.

As Simon and Zame (1990) have pointed out, endogenizing the tie-breaking rule simplifies the existence problem considerably. Due to this simplification, our existence result enables us to dispense with several of the assumptions made in Monteiro and 
Page (2008a), obtaining as a result a richer economic model that allows for: (1) a more general contract space, allowing, in particular, for non-exclusive contracts, (2) more general payoff functions for the principals that, in particular, can depend on the menu of contracts being offered and (3) more general utility functions for the agent.

Furthermore, at a technical level, our result dispenses with the equicontinuity assumption on the agent's utility function used by Monteiro and Page (2008a). However, in contrast with their result, ours requires continuous payoff functions for the principals whereas the existence result of Monteiro and Page (2008a) allows for upper semi-continuous payoff functions that are quasi-linear.

Our existence result can also be used to establish the existence of equilibria in several common agency problems considered in the literature under general assumptions. This is explicitly done for the retail market model of Martimort and Stole (2003) and the lobby problem of Martimort and Semenov (2006).

In summary, our contributions are: (1) to show that the approach of Simon and Zame (1990) is quite appropriate to address the existence of equilibrium in common agency games, (2) to generalize the main result of Simon and Zame (1990), and (3) to obtain a general existence result for common agency games.

The paper is organized as follows. The model is presented in Section 2. In Section 3, we provide the generalization of Simon and Zame's Theorem, establish our existence result and present two applications of our main result. Section 4 concludes. The proof of some results are in the Appendix.

\section{Menu Games}

Consider a game with $m$ principals who can offer contracts to a single agent. The set of contracts that principal $i$ can offer is denoted by $K_{i}$ and we assume that:

Assumption $1 K_{i}$ is a compact metric space.

Each principal offers a menu of contracts to the agent. A menu of contracts for principal $i \in I=\{1, \ldots, m\}$ is just a nonempty closed subset $C_{i}$ of $K_{i}$. 
In Martimort and Stole (2002) for example, the set of contracts that a principal can offer equals the set of probability measures over the allocations controlled by him. Under the assumption that the set of these allocations is finite, it follows that each principal's contract space is compact. They allow each principal $i$ to offer a mechanism to the agent, consisting of a message space $\mathcal{M}_{i}$ and an outcome function $g_{i}: \mathcal{M}_{i} \rightarrow K_{i}$. If $\mathcal{M}_{i}$ is compact and $g_{i}$ is continuous, then the menu $g_{i}\left(\mathcal{M}_{i}\right)$ induced by $\left(\mathcal{M}_{i}, g_{i}\right)$ is a closed subset of $K_{i}$. Thus, in this setting, assuming that menus are closed subsets of $K_{i}$ amounts to assuming that principals use mechanisms with compact message spaces and continuous outcome functions.

Let $P_{i}$ be the collection of all nonempty, closed subsets of $K_{i}$. It is well known that $P_{i}$ is a compact metric space when endowed with the Hausdorff metric. Let $P=P_{1} \times \cdots \times P_{m}$ and $C=\left(C_{1}, \ldots, C_{m}\right)$ denote a profile of menus.

Let $\mathbb{K}$ denote the pure action space of the agent and $k$ denote a generic element of $\mathbb{K}$. We assume that:

Assumption $2 \mathbb{K}$ is a compact metric space.

There are two particular cases for $\mathbb{K}$ in which we are interested. One, considered in Page and Monteiro (2003), is $\mathbb{K}^{P M}=\left\{(i, f) \in I \times \cup_{i=1}^{m} K_{i}: f \in K_{i}\right\}$, where $I=\{1, \ldots, m\}$. Here, the agent chooses the principal with whom she wishes to contract and chooses one contract from this principal. Implicitly, the assumption is that contracts are exclusive.

A second particular case, considered in Martimort and Stole (2002), is $\mathbb{K}^{M S}=$ $K_{1} \times \cdots \times K_{m}$. In this case, contracts are not exclusive, and so the agent can choose a contract from each principal.

These two cases can be combined in a hybrid model in which the agent chooses an exclusive principal within several sub-groups of principals. For example, the agent may have to choose one exclusive electricity company out of two such companies but chooses to contract with all cell phone companies. Formally, the hybrid model is defined by a partition $\left\{I_{n}\right\}_{n=1}^{N}$ of $I$ and by $\mathbb{K}^{H}=\prod_{n=1}^{N}\left\{(i, f) \in I_{n} \times \cup_{i \in I_{n}} K_{i}: f \in\right.$ 
$\left.K_{i}\right\}{ }^{4}$ It is clear that $\mathbb{K}^{P M}, \mathbb{K}^{M S}$ and $\mathbb{K}^{H}$ are compact.

The agent's payoff depends on her type. The set of agent's types is denoted by $T$ and we assume that:

Assumption $3 \mathrm{~T}$ is a Polish space, i.e., $T$ is a complete separable metric space.

We endow $T$ with its Borel $\sigma$-algebra and let $\mu$ describe the probability measure on the set of types. ${ }^{5}$ The agent's utility function is $v: T \times \mathbb{K} \rightarrow \mathbb{R}$ and we assume:

Assumption $4 v$ is a Carathéodory function. ${ }^{6}$

The agent's problem is as follows. Knowing $t \in T$ and given a menu profile $C$ offered by the principals, she is permitted to randomize over her actions by choosing a Borel probability measure on $\mathbb{K}$, i.e., a behavioral strategy. We let $\Delta(\mathbb{K})$ denote the space of all such probability measures. The set of available behavioral strategies is described by a nonempty compact convex set $\varphi(t, C) \subseteq \Delta(\mathbb{K})$. Furthermore, we assume that:

Assumption 5 The correspondence $\varphi: T \times P \rightrightarrows \Delta(\mathbb{K})$ is continuous.

The idea behind the constraint correspondence $\varphi$ is that the agent can choose only from among the contracts being offered, i.e., she can only choose a contract $f_{i} \in C_{i}$ from principal $i$. Therefore, we have three possible specifications for $\varphi$ corresponding to the above particular cases for $\mathbb{K}$ :

$$
\varphi^{P M}(t, C)=\left\{\lambda \in \Delta\left(\mathbb{K}^{P M}\right): \lambda\left(\cup_{i=1}^{m}\left(\{i\} \times C_{i}\right)\right)=1\right\}
$$

in the exclusivity case,

$$
\varphi^{M S}(t, C)=\left\{\lambda \in \Delta\left(\mathbb{K}^{M S}\right): \lambda(C)=1\right\}
$$

\footnotetext{
${ }^{4}$ In all the above models, we can let some $f \in K_{i}$ denote no contracting, following Page and Monteiro (2003).

${ }^{5}$ Throughout the paper, we endow all metric spaces we consider with their Borel $\sigma$-algebra. Therefore, we abbreviate Borel-measurable by measurable.

${ }^{6}$ If $(S, \Sigma)$ is a measurable space, $X$ and $Y$ are topological spaces and $f: S \times X \rightarrow Y$ is a function, then $f$ is a Carathéodory function if it is measurable in $s$ and continuous in $x$. Note that a Carathéodory function is jointly measurable (see Aliprantis and Border (1999, Lemma 4.50, p. 151)).
} 
in the non-exclusivity case and

$$
\varphi^{H}(t, C)=\left\{\lambda \in \Delta\left(\mathbb{K}^{H}\right): \lambda\left(\prod_{n=1}^{N}\left(\cup_{i \in I_{n}}\left(\{i\} \times C_{i}\right)\right)\right)=1\right\}
$$

in the hybrid case. Lemma 5 in Appendix shows that $\varphi^{P M}, \varphi^{M P}$ and $\varphi^{H}$ are continuous with nonempty, convex and compact values.

Hence, given $t \in T$ and $C \in P$, the agent's problem is

$$
\max _{\lambda \in \varphi(t, C)} \int_{\mathbb{K}} v(t, k) \mathrm{d} \lambda(k) .
$$

Let $\Lambda: T \times P \rightrightarrows \Delta(\mathbb{K})$ denote the correspondence of optimal choices. A strategy for the agent is then a measurable function $\sigma: T \times P \rightarrow \Delta(\mathbb{K})$, and, clearly, $\sigma$ is an optimal strategy if and only if it is a selection of $\Lambda$.

We now turn to the principals' problem. Principals choose simultaneously. For all $i \in I$, principal $i$ 's choice set is $\Delta\left(P_{i}\right)$, the set of mixed strategies on $P_{i}$, and his payoff function is denoted by $\pi_{i}: T \times P \times \mathbb{K} \rightarrow \mathbb{R}$. We assume:

Assumption $6 \pi_{i}$ is an integrably bounded Carathéodory function, i.e., $t \mapsto \pi_{i}(t, C, k)$ is measurable for all $(C, k) \in P \times \mathbb{K},(C, k) \mapsto \pi_{i}(t, C, k)$ is continuous for all $t \in T$, and there exists an integrable function $b_{i}: T \rightarrow \mathbb{R}$ such that $\left|\pi_{i}(t, C, k)\right| \leq b_{i}(t)$ for all $(t, C, k) \in T \times P \times \mathbb{K}$.

Note that in the above formalization we allow each principal's payoff to depend on the type $t$ of the agent, on the choice $k$ of the agent and also on the contracts $C$ that he and the other principals have offered. A possible justification for the dependence of a principal's payoff on the contracts being offered include the cost of writing each contract (so the payoff of principal $i$ decreases with the cardinality of $C_{i}$ ). It is important to note that the delegation principle of Martimort and Stole (2002) and Page and Monteiro (2003) extends to this more general framework, as can be easily verified, implying that menu games are still appropriate for analyzing common agency problems in which such dependence holds.

If the principals offer a menu $C=\left(C_{1}, \ldots, C_{m}\right) \in P$ and the agent uses a strategy $\sigma: T \times P \rightarrow \Delta(\mathbb{K})$, then principal $i$ 's payoff is

$$
F_{i}(t, C ; \sigma)=\int_{\mathbb{K}} \pi_{i}(t, C, k) \mathrm{d} \sigma(k \mid t, C) .
$$


Since $\sigma$ is measurable, then so is the real-valued function $F_{i}$ on $T \times P$. Finally, if principals choose strategies $\alpha=\left(\alpha_{1}, \ldots, \alpha_{m}\right)$ and the agent chooses a strategy $\sigma$,

$$
F_{i}(\alpha ; \sigma)=\int_{P} \int_{T} F_{i}(t, C ; \sigma) \mathrm{d} \mu(t) \mathrm{d} \alpha(C)
$$

denotes principal $i$ 's payoff.

A menu game is then described by $G=\left(I,\left(K_{i}, \pi_{i}\right)_{i \in I}, \mathbb{K}, T, \mu, v, \varphi\right)$. Furthermore, we use $G^{P M}, G^{M S}$ and $G^{H}$ to denote particular menu games for the corresponding choices of $\mathbb{K}$ and $\varphi$ mentioned above.

As in Martimort and Stole (2002), we consider the subgame perfect equilibria (SPE) of a menu game $G$. A strategy $(\alpha, \sigma)$ is a SPE of a menu game $G$ if and only if

1. $\sigma$ is a measurable selection of $\Lambda$ and

2. $F_{i}(\alpha ; \sigma) \geq F_{i}\left(\alpha_{i}^{\prime}, \alpha_{-i} ; \sigma\right)$ for all $i \in I$ and $\alpha_{i}^{\prime} \in \Delta\left(P_{i}\right)$.

Thus, in a SPE of $G$, the agent optimizes for all possible types and menus offered, and each principal optimizes given the strategy of the other principals and the strategy of the agent.

Equivalently, we can describe a $\mathrm{SPE}$ in the following way: a strategy $(\alpha, \sigma)$ is a SPE if $\sigma$ is an optimal strategy for the agent and $\alpha$ is a Nash equilibrium of the (possibly discontinuous) normal-form game $\left(P_{i}, F_{i}(\cdot ; \sigma)\right)_{i \in I}$ induced by $\sigma$.

\section{Existence of Equilibrium}

Our main result is the following existence theorem.

Theorem 1 A SPE exists for all menu games $G$ satisfying assumptions 1 - 6 .

Since the frameworks of Page and Monteiro (2003) and Martimort and Stole (2002) are particular cases of ours, we have the following corollary.

Corollary 1 All menu games $G^{H}, G^{P M}$ and $G^{M S}$ satisfying assumptions 1 - 6 have a SPE. 
In order to establish Theorem 1 we first generalize the theorem in Simon and Zame (1990) by allowing the payoff correspondence to depend on the agent's type. We then use this result to prove the existence of a subgame perfect equilibrium in any continuous menu game. This last argument uses Lemma 2, below, which shows how to obtain a subgame perfect equilibrium from a solution of a (generalized) game with an endogenous sharing rule.

\subsection{A Generalization of Simon and Zame's Theorem}

A generalized game with an endogenous sharing rule is an $m+3$-tuple $G=\left(P_{1}, \ldots, P_{m}, T, \mu, Q\right)$ where $P_{i}$ is a compact metric space for all $i, T$ is a Polish space, $\mu$ is a measure defined on the Borel measurable subsets of $T$ and $Q: T \times P \rightrightarrows \mathbb{R}^{m}$ is measurable, integrably bounded (i.e., there exists a $\mu$-integrable function $b: T \rightarrow \mathbb{R}$ such that $\|u\| \leq b(t)$ for all $u \in Q(t, C)$ and for all $(t, C) \in T \times C)$, has nonempty, convex and compact values and is upper hemi-continuous in $C$ for all $t \in T$ (i.e., $C \mapsto Q(t, C)$ is upper hemi-continuous for all $t \in T)$.

A solution for $G$ is a pair $(q, \alpha)$ such that $q$ is a measurable selection of $Q,{ }^{7}$ $\alpha_{i} \in \Delta\left(P_{i}\right)$ and

$$
\int_{P} \int_{T} q_{i}(t, C) \mathrm{d} \mu(t) \mathrm{d} \alpha(C) \geq \int_{P} \int_{T} q_{i}(t, C) \mathrm{d} \mu(t) \mathrm{d}\left(\beta_{i} \times \alpha_{-i}\right)(C)
$$

for all $i$ and all $\beta_{i} \in \Delta\left(P_{i}\right)$.

Theorem 2 A solution exists for all generalized games with an endogenous sharing rule.

The proof of Theorem 2 follows closely the one in Simon and Zame (1990) and is presented in Appendix A.1.

\footnotetext{
${ }^{7} \mathrm{~A}$ function $q: T \times P \rightarrow \mathbb{R}^{m}$ is a measurable selection of $Q$ if $q$ is measurable and $q(t, C) \in Q(t, C)$ for all $(t, C) \in T \times P$.
} 


\subsection{Proof of Theorem 1}

The proof of Theorem 1 proceeds as follows: first, we define a generalized game with an endogenous sharing rule, essentially, defining the payoff correspondence by composing principals' payoff functions with the optimal choice correspondence of the agent. We then use Theorem 2 to obtain a solution to that generalized game with an endogenous sharing rule. Then, we use Lemma 2 to show that the measurable selection from the payoff correspondence can be written as the composition between the principals' payoff function and a measurable selection from the agent's optimal choice correspondence (i.e., an optimal strategy for the agent). Finally, we show that this strategy together with the principals' strategies that are part of the solution for the generalized game with an endogenous sharing rule form a subgame perfect equilibrium strategy.

Let $h: T \times P \times \Delta(\mathbb{K}) \rightarrow \mathbb{R}^{m}$ be defined by

$$
h(t, C, \lambda)=\int_{\mathbb{K}} \pi(t, C, k) \mathrm{d} \lambda(k) .
$$

Note that if $\sigma: T \times P \rightarrow \Delta(\mathbb{K})$ is a strategy for the agent, then $F(t, C ; \sigma)=$ $h(t, C, \sigma(t, C))$ for all $t \in T$ and $C \in P$. Also, note that $(C, \lambda) \mapsto h(t, C, \lambda)$ is continuous and $t \mapsto h(t, C, \lambda)$ is measurable. Hence, by Aliprantis and Border (1999, Lemma 4.50, p. 151$), h$ is (jointly) measurable since $P \times \Delta(\mathbb{K})$ is a compact metric space.

Furthermore, letting $S=T \times P, h: S \times \Delta(\mathbb{K}) \rightarrow \mathbb{R}$ is measurable in $s=(t, C)$ and continuous in $\lambda$, and so a Carathéodory function (although $h$ satisfies additional properties, this suffices to prove Theorem 1). Define $Q: T \times P \rightrightarrows \mathbb{R}^{m}$ by

$$
Q(t, C)=\{h(t, C, \lambda): \lambda \in \Lambda(t, C)\}
$$

Lemma 1 The correspondence $\Lambda$ is measurable and has compact values. The correspondence $Q$ is measurable, integrably bounded, upper hemi-continuous in $C$ for all $t \in T$ and has nonempty, convex and compact values.

It follows by Lemma 1 that $G=\left(P_{1}, \ldots, P_{m}, T, \mu, Q\right)$ is a generalized game with 
an endogenous sharing rule. Hence, by Theorem 2 , there exists a solution $(q, \alpha)$ for G.

In order to obtain a subgame perfect equilibrium from the solution $(q, \alpha)$, we use the following lemma.

Lemma 2 Let $S$ be a measurable space, $X$ be a compact metric space, $g: S \times$ $X \rightarrow \mathbb{R}^{m}$ a Carathéodory function and $\Theta: S \rightrightarrows X$ a compact valued, measurable correspondence.

If $Q: S \rightrightarrows \mathbb{R}^{m}$ is defined by

$$
Q(s)=\{g(s, x): x \in \Theta(s)\}
$$

for all $s \in S$ and $q$ is a measurable selection of $Q$, then there exists a measurable selection $\alpha$ of $\Theta$ such that $q(s)=g(s, \alpha(s))$ for all $s \in S$.

This Lemma follows from Furukawa (1972, Lemma 4.6) and Aliprantis and Border (1999, Theorem 17.10, p. 565) .

Since $T \times P$ is a measurable space, $\Delta(\mathbb{K})$ is a compact metric space and $\Lambda$ is compact valued and measurable, then by Lemma 2 , there exists a measurable selection $\sigma$ from $\Lambda$ such that $q(t, C)=h(t, C, \sigma(t, C))$ for all $t \in T$ and $C \in P$. Hence,

$$
\begin{aligned}
& F_{i}(\alpha ; \sigma)=\int_{P} \int_{T} h_{i}(t, C, \sigma(t, C)) \mathrm{d} \mu(t) \mathrm{d} \alpha(C)= \\
& \int_{P} \int_{T} q_{i}(t, C) \mathrm{d} \mu(t) \mathrm{d} \alpha(C) \geq \int_{P} \int_{T} q_{i}(t, C) \mathrm{d} \mu(t) \mathrm{d}\left(\beta_{i} \times \alpha_{-i}\right)(C)= \\
& \int_{P} \int_{T} h_{i}(t, C, \sigma(t, C)) \mathrm{d} \mu(t) \mathrm{d}\left(\beta_{i} \times \alpha_{-i}\right)(C)=F_{i}\left(\beta_{i}, \alpha_{-i} ; \sigma\right)
\end{aligned}
$$

for all $i$ and all $\beta_{i} \in \Delta\left(P_{i}\right)$. It then follows that $(\alpha, \sigma)$ is a SPE of $G$.

\subsection{Two Examples}

In this subsection, we derive from Theorem 1 a general existence result for the retail market and lobby models of Martimort and Stole (2003) and Martimort and Semenov (2006), respectively. 


\subsubsection{Retail Market Game}

In this subsection, we consider a generalized version of Martimort and Stole (2003). The principals are thought to be retailers that sell perfect substitutes in a final market, while the agent is the single supplier of the intermediate goods needed to produce the final good.

Assume that there are $l$ intermediate goods and let the contract space be a compact subset $K$ of $\mathbb{R}^{l+1}$ with the following interpretation: the contract $(y, d)$, with $y \in \mathbb{R}^{l}$ and $d \in \mathbb{R}$ specifies that the agent must produce the vector of quantities $y=\left(y_{1}, \ldots, y_{l}\right)$ of the $l$ intermediate goods, receiving $d$ dollars in compensation.

Assume that there are $m$ principals with $K_{i}=K$ for all $i=2, \ldots, m, K_{1}=$ $\left\{k_{n}, k_{c}\right\}$ with $k_{n} \neq k_{c}$ and let $\mathbb{K}=\mathbb{K}^{M S}$. The choice of $k_{c} \in K_{1}$ is interpreted as the decision to contract by the agent, while $k_{n}$ means that the agent does not contract with any principal. Thus, we are considering the intrinsic common agency problem. Also, if $k \in K$, then $k=\left(k_{1}, \ldots, k_{m}\right)$ and $k_{i}=\left(y_{i}, d_{i}\right)$ for all $i \geq 2$.

Let $Y$ be the projection of $K$ onto the first $l$ coordinates. Principal $i, i \geq 2$, uses the $l$ intermediate goods to produce $p$ final goods according to the continuous production function $g_{i}: Y \rightarrow \mathbb{R}^{p}$. Let $X=\sum_{i=2}^{m} g_{i}(Y)$ and let $P: X \rightarrow \mathbb{R}^{p}$ be the continuous inverse demand function of the $p$ final goods. Principals' payoffs are defined by

$$
\pi_{1}(t, C, k)=\left\{\begin{array}{lll}
1 & \text { if } & C_{1}=K_{1}=\left\{k_{n}, k_{c}\right\} \\
0 & \text { if } & \text { otherwise }
\end{array}\right.
$$

And for $i \geq 2$ :

$$
\pi_{i}(t, C, k)=\left\{\begin{array}{lll}
P\left(\sum_{j=2}^{m} g_{i}\left(y_{i}\right)\right) \cdot g_{i}\left(y_{i}\right)-d_{i} & \text { if } \quad k_{1}=k_{c} \\
0 & \text { if } \quad k_{1}=k_{n}
\end{array}\right.
$$

The agent's technology is described by an integrably bounded Carathéodory cost function $c: T \times Y \rightarrow \mathbb{R}$ and her utility function is

$$
v(t, k)=\left\{\begin{array}{lll}
\sum_{i=2}^{m} d_{i}-g\left(t, \sum_{i=2}^{m} y_{i}\right) & \text { if } & k_{1}=k_{c} \\
0 & \text { if } & k_{1}=k_{n} .
\end{array}\right.
$$


Therefore, we allow for multi-goods, in both the final and the intermediate markets and more general cost/production functions, which, in particular, do not have to be differentiable. Despite these generalizations, it follows from Theorem 1 that this model has a SPE.

\subsubsection{Lobbying Game}

Consider $m$ lobbying groups (principals) that want to influence a decision-maker (agent), who chooses a policy variable $q \in K_{q}$ where $K_{q}$ is a compact subset of $\mathbb{R}^{L}$. The variable $q$ can be interpreted as a level of vector of $L$ public goods, a vector of $L$ regulated prices, etc. Each principal chooses a vector of contributions $\theta \in K_{\theta}$, a compact subset of $\mathbb{R}_{+}^{L}$.

We first consider the case where the principals make contributions for each of the $L$ policy variables. A contract is then a pair $(q, \theta) \in K_{q} \times K_{\theta}$ : for all $l=1, \ldots, L$, if the agent chooses policy $q_{l}$, then the principal pays contribution $\theta_{l}$. Thus, the contract space for principal $i$ is $K_{i}=K_{q} \times K_{\theta}$ for all $i$.

Let $\mathcal{J}$ be the set of all subsets of $\{1,2, \ldots, m\}$ (not necessarily nonempty). The pure action space of the agent is $\mathbb{K}=K_{q} \times K_{\theta}^{m} \times \mathcal{J}^{L}$ with the following interpretation: if the agent chooses $(q, \theta, J)=\left(q, \theta_{1}, \ldots, \theta_{m}, J_{1}, \ldots, J_{L}\right)$, it means that she will implement policy $q$ and, for all $l=1, \ldots, L$, accept contributions $\theta_{i, l}$ for all $i \in J_{l}$.

However, the agent can only accept contributions corresponding to the policy level she chooses. This is used to define her constraint correspondence $\varphi: T \times P \rightrightarrows \Delta(\mathbb{K})$ as follows: for all $l=1, \ldots, L$, let $\operatorname{proj}_{l}: \mathbb{R}^{L} \rightarrow \mathbb{R}$ denote the projection onto the $l$ th coordinate and define

$$
\begin{aligned}
\varphi(t, C)= & \{\lambda \in \Delta(\mathbb{K}): \lambda(\{(q, \theta, J) \in \mathbb{K}: \text { for all } l=1, \ldots, L, \\
& \left.\left.\left.\left(q_{l}, \theta_{i, l}\right) \in \operatorname{proj}_{l}\left(C_{i}\right) \text { for all } i \in J_{l}\right\}\right)=1\right\} .
\end{aligned}
$$

The agent's utility function is $v: T \times \mathbb{K} \rightarrow \mathbb{R}$ defined by:

$$
v(t, q, \theta, J)=V\left(\sum_{l=1}^{L} \sum_{i \in J_{l}} \theta_{i, l}-C(t, q)\right),
$$

where $C: T \times K_{q} \rightarrow \mathbb{R}$ is a continuous cost function, interpreted as an opportunity cost and $V: \mathbb{R} \rightarrow \mathbb{R}$ is the agent's continuous money utility function. 
For each $i=1, \ldots, m$, principal $i$ 's payoff function $\pi_{i}: T \times \mathbb{K} \rightarrow \mathbb{R}$ is given by

$$
\pi_{i}(t, q, \theta, J)=U_{i}\left(R_{i}(t, q)-\sum_{l: i \in J_{l}} \theta_{i, l}\right),
$$

where the continuous function $R_{i}: T \times K_{q} \rightarrow \mathbb{R}$ captures the benefit he obtains from policy $q$ and $U_{i}$ is his continuous money utility function.

Finally, we consider the case where principals make a contribution for a global policy. In this case, $K_{\theta}$ is a compact subset of $\mathbb{R}_{+}$. The contract space is still $K_{i}=K_{q} \times K_{\theta}$, with the interpretation that if the agent chooses $q \in K_{q}$, then the principal pays $\theta \in K_{\theta}$. Since the agent can no longer accept a contribution for each policy variable, we let $\mathbb{K}=K_{q} \times K_{\theta}^{m} \times \mathcal{J}$.

The agent's constraint correspondence is

$$
\varphi(t, C)=\left\{\lambda \in \Delta(\mathbb{K}): \lambda\left(\left\{(q, \theta, J) \in \mathbb{K}:\left(q, \theta_{i}\right) \in C_{i} \text { for all } i \in J\right\}\right)=1\right\}
$$

and her utility function is

$$
v(t, q, \theta, J)=V\left(\sum_{i \in J} \theta_{i}-C(t, q)\right) .
$$

Finally, principal $i$ 's payoff function is

$$
\pi_{i}(t, q, \theta, J)= \begin{cases}U_{i}\left(R_{i}(t, q)-\theta_{i}\right) & \text { if } \quad i \in J, \\ U_{i}(0) & \text { if } \quad i \in J^{c}\end{cases}
$$

It follows from Theorem 1 that this game has a SPE. It is worth noting that this model is a generalized version of a model suggested by Martimort and Semenov (2006). This is the case since we allow for many policy variables, more than two principals, risk aversion and more general payoff functions.

\section{Conclusions}

We have shown that a SPE exists in all menu games. Compared with the results of Page and Monteiro (2003) and Monteiro and Page (2008a), our existence theorem has the advantage of allowing for a richer economic model, dispensing, in particular, 
with the exclusivity and the no-fixed-cost assumptions made in those papers. Our approach relies heavily on the ideas of Simon and Zame (1990). In fact, the proof of our existence result is straightforward once we extend their theorem to the case in which the principals' payoff correspondence depends on the agent's type.

\section{A Appendix}

In the appendix, we prove Theorem 2, and Lemmas 1 and 2. Also, we prove that the correspondences $\varphi^{P M}, \varphi^{M S}$ and $\varphi^{H}$ are continuous with nonempty, convex, compact values.

\section{A.1 Proof of Theorem 2}

We start to note that we may assume that $Q$ is bounded. ${ }^{8}$ In fact, let $b: T \rightarrow \mathbb{R}$ be such that $\|u\|=\max _{1 \leq i \leq m}\left|u_{i}\right| \leq b(t)$ for all $u=\left(u_{1}, \ldots, u_{m}\right) \in Q(t, C)$ and $(t, C) \in$ $T \times P$ and define, for each $t \in T, \gamma(t)=1+b(t)$. Transform the payoff correspondence $Q$ by defining $\widetilde{Q}(t, C)=\left\{\widetilde{u} \in \mathbb{R}^{m}: \gamma(t) \widetilde{u} \in Q(t, C)\right\}$ for all $(t, C) \in T \times P$. The correspondence $\widetilde{Q}$ is bounded: for all $(t, C) \in T \times P$ and $\widetilde{u} \in \widetilde{Q}(t, C)$,

$$
\|\widetilde{u}\| \leq \sum_{i=1}^{m}\left|\widetilde{u}_{i}\right|=\sum_{i=1}^{m} \frac{\left|\gamma(t) \widetilde{u}_{i}\right|}{\gamma(t)} \leq \sum_{i=1}^{m} \frac{\gamma(t)}{\gamma(t)}=m .
$$

Furthermore, $\widetilde{Q}$ inherits all the other properties of $Q$. Transform the measure $\mu$ by defining $A=\left[\int_{T} \gamma(t) \mathrm{d} \mu(t)\right]^{-1}>0$ and $\widetilde{\mu}(B)=A \int_{B} \gamma(t) \mathrm{d} \mu(t)$ for all measurable subset $B$ of $T$. We then obtain that, for any measurable selection $\widetilde{q}$ of $\widetilde{Q}$ and any $C \in P$,

$$
\int_{T} \widetilde{q}_{i}(t, C) \mathrm{d} \widetilde{\mu}(t)=\int_{T} \frac{q_{i}(t, C)}{\gamma(t)} A \gamma(t) \mathrm{d} \mu(t)=A \int_{T} q_{i}(t, C) \mathrm{d} \mu(t),
$$

where $q(t, C)=\gamma(t) \widetilde{q}(t, C) \in Q(t, C)$ for all $(t, C) \in T \times P$. Therefore, if $(\widetilde{q}, \alpha)$ is a solution of the transformed game $\widetilde{G}=\left(P_{1}, \ldots, P_{m}, T, \widetilde{\mu}, \widetilde{Q}\right)$, then $(q, \alpha)$ is a solution of the original game $G$, where $q(t, C)=\gamma(t) \widetilde{q}(t, C)$ for all $(t, C) \in T \times P$.

\footnotetext{
${ }^{8}$ We are thankful to an associate editor and an anonymous referee for suggesting to us this argument.
} 
As a consequence of the above argument, we may assume that $Q$ is bounded. We note that, in this case, we may assume that there exists $w, W>0$ such that $w \leq u_{i} \leq W$ for all $i=1, \ldots, m, u=\left(u_{1}, \ldots, u_{m}\right) \in Q(t, C)$ and $(t, C) \in T \times P$. In fact, if $B \in \mathbb{R}$ is such that $\|u\|=\max _{1 \leq i \leq m}\left|u_{i}\right| \leq B$ for all $u=\left(u_{1}, \ldots, u_{m}\right) \in Q(t, C)$ and $(t, C) \in T \times P$, define $\hat{Q}(t, C)=Q(t, C)+(B, \ldots, B)+w=\left\{\hat{u} \in \mathbb{R}^{m}: \hat{u}=\right.$ $u+(B+w, \ldots, B+w), u \in Q(t, C)\}$ for all $(t, C) \in T \times P$. Letting $W=w+2 B$, we obtain that $w \leq u_{i} \leq W$ for all $i$ and all $u \in \hat{Q}(t, C)$ and $\hat{G}=\left(P_{1}, \ldots, P_{m}, T, \mu, \hat{Q}\right)$ is a generalized game with an endogenous sharing rule. Furthermore, it is easy to see that if $(\hat{q}, \alpha)$ is a solution for $\hat{G}$ and $q: T \times P \rightarrow \mathbb{R}^{m}$ is defined by $q(t, C)=$ $\hat{q}(t, C)-(B+w, \ldots, B+w)$ for all $(t, C) \in T \times P$, then $(q, \alpha)$ is a solution for $G$.

Due to the above, we assume from now on that there exists $w, W>0$ such that $w \leq u_{i} \leq W$ for all $i=1, \ldots, m, u=\left(u_{1}, \ldots, u_{m}\right) \in Q(t, C)$ and $(t, C) \in T \times P$.

Our proof of Theorem 2 from now on follows the one in Simon and Zame (1990). Indeed, we start by modifying their Lemma 2 and then proceed by adapting the six steps of their proof to our setting.

Both their Lemma 2 and our version of it applies to vector-valued measures defined as follows. If $S$ is a Polish space, $\psi$ is a probability measure on $S$, and $q$ is a measurable, $\psi$-integrably bounded function from $S$ into $\mathbb{R}^{m}$, define $q \psi \in \Delta(S)$ by

$$
q \psi(B)=\int_{B} q \mathrm{~d} \psi
$$

for all measurable subsets $B$ of $S$.

A particular case is when $S=T \times P$ and $\psi=\mu \times \alpha$ for some $\alpha \in \Delta(P)$. In this case, let $q: T \times P \rightarrow \mathbb{R}^{m}$ be $\mu$-integrably bounded and measurable and define $\hat{q}: P \rightarrow \mathbb{R}^{m}$ by

$$
\hat{q}(C)=\int_{T} q(t, C) \mathrm{d} \mu(t)
$$

The following lemma considers the above special case and establishes a property of $q(\mu \times \alpha)$ that is useful in our version of Simon and Zame's Lemma 2. It uses the following notation: if $X$ and $Y$ are metric spaces and $\nu$ is a measure on $X \times Y, \nu_{Y}$ denotes the marginal distribution of $\nu$ on $Y$. 
Lemma 3 If $q: T \times P \rightarrow \mathbb{R}^{m}$ is bounded and measurable, then $\hat{q}$ is measurable and

$$
\hat{q} \alpha=q(\mu \times \alpha)_{P} .
$$

Proof. Since $q$ is bounded and measurable, the integral exists. The measurability of $\hat{q}$ follows from Fubini's Theorem (see Aliprantis and Border (1999, Theorem 11.26, p. 411)).

We turn to the second claim. Let $B$ be a measurable subset of $P$. It follows that

$$
\begin{aligned}
& \hat{q} \alpha(B)=\int_{B} \hat{q} \mathrm{~d} \alpha=\int_{B} \int_{T} q \mathrm{~d} \mu \mathrm{d} \alpha= \\
& \int_{T \times B} q \mathrm{~d}(\mu \times \alpha)=q(\mu \times \alpha)(T \times B)=q(\mu \times \alpha)_{P}(B) .
\end{aligned}
$$

Thus, the lemma follows.

After these preliminaries, we turn to our version of Lemma 2 in Simon and Zame (1990). There, we allow for the case where $S$ is the product of a Polish space (i.e., a complete separable metric space) and a compact metric space and $Q$ is measurable but only upper hemi-continuous in the second variable. However, we assume that all the measures involved are finite. Recall that a vector measure on a metric space $X$ is a countably additive set function $\tau$ from the Borel $\sigma$-algebra of $X$ into a Banach space $Y$. A sequence of vector measures $\left\{\tau_{n}\right\}_{n}$ converge weakly to a vector measure $\tau$ if $\int_{X} f \mathrm{~d} \tau=\lim _{n} \int_{X} f \mathrm{~d} \tau_{n}$ for all bounded, continuous functions $f: X \rightarrow Y^{9}$

Lemma 4 Let $\left\{\nu_{n}\right\}$ be a sequence of probability measures on $P$ converging weakly to $\nu$ and let $Q: T \times P \rightrightarrows \mathbb{R}^{m}$ be a bounded, measurable correspondence, upper hemicontinuous in $C$ for all $t \in T$ and with compact, convex, nonempty values. For each $n$, let $q_{n}$ be a measurable selection from $Q$.

If the sequence $\left\{q_{n}\left(\mu \times \nu_{n}\right)\right\}$ of vector-valued measures converges weakly to a vectorvalued measure $\xi$, then there exists a measurable selection $q$ from $Q$ such that $\xi=$ $q(\mu \times \nu)$.

\footnotetext{
${ }^{9}$ See Diestel and Uhl (1977) for more details about vector measures and März and Shorrt (1994) for weak convergence of vector measures.
} 
Proof. Note that $\left\{\mu \times \nu_{n}\right\}$ converges weakly to $\{\mu \times \nu\}$ by Hildenbrand (1974, Theorem 27, pg. 49). Therefore, the boundedness of $Q$ implies, as in Simon and Zame (1990, Lemma 2), that there exists a measurable function $q: T \times P \rightarrow \mathbb{R}^{m}$ such that $\xi=q(\mu \times \nu)$.

Let $H=\{(t, C) \in T \times P: q(t, C) \notin Q(t, C)\}$. Since both $q$ and $Q$ are measurable, then $H$ is measurable. In fact, let $S=T \times P$ for convenience, $f=\left(i d_{S}, q\right)$ and $\delta$ : $S \times \mathbb{R}^{m} \rightarrow \mathbb{R}$ be defined by $\delta(s, x)=\max _{z \in Q(s)}\|x-z\|$. Clearly, $f$ is measurable since $q$ is also measurable. Since $Q$ is measurable, then $Q$ is weakly measurable by Theorem 17.2 in Aliprantis and Border (1999, p. 559). Then, $\delta$ is a Carathéodory function by Theorem 17.5 in Aliprantis and Border (1999, p. 562) and thus measurable since $\mathbb{R}^{m}$ is separable (see Aliprantis and Border (1999, Lemma 4.50, p.151)). It follows that the function $g: S \rightarrow \mathbb{R}$ defined by $g=\delta \circ f$ is measurable and that $H=\{s \in S: g(s)>0\}$ is a measurable subset of $S$.

In order to show that $q(t, C) \in Q(t, C) \mu \times \nu$-a.e., consider the following. Let $t \in T$ and $H_{t}=\{C \in P:(t, C) \in H\}$. Since $H$ is a measurable subset of $T \times P$, then $H_{t}$ is a measurable subset of $P$ (see Aliprantis and Border (1999, Lemma 4.45, p. 148)). Since $P$ is compact and $c \mapsto Q(t, c)$ is upper hemi-continuous, it follows by the arguments of Simon and Zame that $\nu\left(H_{t}\right)=0$. Thus,

$$
\mu \times \nu(H)=\int_{T} \nu\left(H_{t}\right) \mathrm{d} \mu(t)=0
$$

by Fubini's Theorem. This completes the proof since we can correct $q$ in $H$, obtaining a function that is still measurable.

We turn to the proof of Theorem 2, showing that the same arguments used by Simon and Zame extend to our setting, with minor changes.

Recall that $w, W>0$ is such that $w \leq u_{i} \leq W$ for all $i=1, \ldots, m, u=$ $\left(u_{1}, \ldots, u_{m}\right) \in Q(t, C)$ and $(t, C) \in T \times P$.

Step 1: Finite approximations. Let $g$ be a measurable selection from $Q$. Recall that $P$ is a compact metric space. As in Simon and Zame (1990), discretize $P$ in order to obtain, for all $r \in \mathbb{N}$, a finite action space $P_{i}^{r}$ for all players $i=1, \ldots, m$ and a 
Nash equilibrium $\left(\alpha_{1}^{r}, \ldots, \alpha_{m}^{r}\right)$ for the normal-form game $G^{r}=\left(P_{1}^{r}, \ldots, P_{m}^{r}, \hat{g}\right)$ (recall that $\hat{g}$ is defined by $\hat{g}(C)=\int_{T} g(t, C) \mathrm{d} \mu(t)$ for all $\left.C \in P\right)$. Let $\alpha^{r}=\alpha_{1}^{r} \times \cdots \times \alpha_{m}^{r}$.

Step 2: Limits. Since $\Delta\left(P_{i}\right)$ is compact, then we may assume that $\left\{\alpha_{i}^{r}\right\}_{r}$ converges for all $i=1, \ldots, m$. Thus, $\left\{\alpha^{r}\right\}_{r}$ converges. Letting $\alpha=\lim _{r} \alpha^{r}$, it follows that $\left\{\mu \times \alpha^{r}\right\}_{r}$ converges to $\mu \times \alpha$.

We claim that $\left\{g\left(\mu \times \alpha^{r}\right)\right\}_{r}$ has a convergent subsequence. Since $g=\left(g_{1}, \ldots, g_{m}\right)$, it suffices to show that $\left\{g_{i}\left(\mu \times \alpha^{r}\right)\right\}_{r}$ has a convergent subsequence for all $i=1, \ldots, m$.

Let $i \in\{1, \ldots, m\}$. For all $r \in \mathbb{N}$, define $\gamma^{r}=\int_{T \times P} g_{i} \mathrm{~d} \mu \times \alpha^{r} \geq w$. Since $g_{i}(t, C) \leq W$ for all $(t, C) \in T \times P$, then $\gamma^{r} \leq W$. Hence, $\left\{\gamma^{r}\right\}_{r}$ is bounded and we may assume that it converges. Let $\gamma=\lim _{r} \gamma^{r} \in[w, W]$.

For all $r \in \mathbb{N}$, define $\breve{g}_{i}^{r}: T \times P \rightarrow \mathbb{R}$ by $\breve{g}_{i}^{r}(t, C)=q_{i}(t, C) / \gamma^{r}$. Then, $\breve{g}_{i}^{r}\left(\mu \times \alpha^{r}\right)$ is a probability measure on $T \times P$. Furthermore, the sequence $\left\{\breve{g}_{i}^{r}\left(\mu \times \alpha^{r}\right)\right\}_{r}$ is tight. Indeed, let $\varepsilon>0$ and let $M>0$ be such that $\breve{g}_{i}^{r}(t, C) \leq M$ for all $(t, C) \in T \times P$ and $r \in \mathbb{R}$ (the existence of $M$ follows because both $g_{i}$ and $\left\{\gamma^{r}\right\}_{r}$ are bounded). Furthermore, let $K$ be a compact subset of $T$ such that $\mu\left(K^{c}\right)<\varepsilon / M$ (note that $\mu$ is tight by Aliprantis and Border (1999, Theorem 10.7, p. 370)). Then, $K \times P$ is a compact subset of $T \times P$ and

$$
\breve{g}_{i}^{r}\left(\mu \times \alpha^{r}\right)\left((K \times P)^{c}\right)=\breve{g}_{i}^{r}\left(\mu \times \alpha^{r}\right)\left(K^{c} \times P\right) \leq M \mu\left(K^{c}\right)<\varepsilon,
$$

establishing that $\left\{\breve{g}_{i}^{r}\left(\mu \times \alpha^{r}\right)\right\}_{r}$ is tight. Therefore, it has a convergent subsequence (see Aliprantis and Border (1999, Theorem 14.22, p. 488)).

For convenience, assume that $\left\{\breve{g}_{i}^{r}\left(\mu \times \alpha^{r}\right)\right\}_{r}$ converges and let $\breve{\nu}=\lim _{r} \breve{g}_{i}^{r}\left(\mu \times \alpha^{r}\right)$. Finally, define $\nu=\gamma \breve{\nu}$. We claim that $\nu=\lim _{r} g_{i}\left(\mu \times \alpha^{r}\right)$. In order to prove this claim, let $f: T \times P \rightarrow \mathbb{R}$ be continuous and bounded. It follows that $\int_{T \times P} f \mathrm{~d} \nu=\gamma \int_{T \times P} f \mathrm{~d} \breve{\nu}$ for all $r \in \mathbb{R}$ (by the definition of $\nu$ ), $\int_{T \times P} f \mathrm{~d} \breve{\nu}=\lim _{r} \int_{T \times P} f \breve{q}_{i}^{r} \mathrm{~d}\left(\mu \times \alpha^{r}\right)$ (since $\left.\breve{\nu}=\lim _{r} \breve{g}_{i}^{r}\left(\mu \times \alpha^{r}\right)\right)$ and so

$$
\begin{aligned}
& \int_{T \times P} f \mathrm{~d}\left(g_{i}\left(\mu \times \alpha^{r}\right)\right)=\int_{T \times P} f g_{i} \mathrm{~d}\left(\mu \times \alpha^{r}\right)= \\
& \gamma^{r} \int_{T \times P} f \breve{g}_{i}^{r} \mathrm{~d}\left(\mu \times \alpha^{r}\right) \rightarrow \gamma \int_{T \times P} f \mathrm{~d} \breve{\nu}=\int_{T \times P} f d \nu .
\end{aligned}
$$

Hence, $\nu=\lim _{r} g_{i}\left(\mu \times \alpha^{r}\right)$. 
We have, therefore, established that, taking a subsequence if necessary, we may assume that $\left\{g\left(\mu \times \alpha^{r}\right)\right\}_{r}$ converges. Let $\xi=\lim _{r} g\left(\mu \times \alpha^{r}\right)$.

Step 3: Selections. By Lemma 4, there exists a measurable selection $q$ from $Q$ such that $\xi=q(\mu \times \alpha)$.

Step 4: Better responses. Recall that $\hat{h}: P \rightarrow \mathbb{R}^{m}$ is defined by $\hat{h}(C)=$ $\int_{T} h(t, C) \mathrm{d} \mu(t)$ for all $\mu$-integrably bounded and measurable functions $h: T \times P \rightarrow$ $\mathbb{R}^{m}$.

By Lemma 3, $\hat{q}$ is measurable and $\alpha_{r}$ is a Nash equilibrium of the normal-form game $\left(P_{1}^{r}, \ldots, P_{m}^{r}, \hat{g}\right)$ for all $r \in \mathbb{N}$. Since $\left\{g\left(\mu \times \alpha^{r}\right)\right\}$ converges to $q(\mu \times \alpha), \hat{q} \alpha=$ $q(\mu \times \alpha)_{P}$ and, similarly, $\hat{g} \alpha^{r}=g\left(\mu \times \alpha^{r}\right)_{P}$ for all $r \in \mathbb{R}$, it follows that $\left\{\hat{g} \alpha^{r}\right\}$ converges to $\hat{q} \alpha$.

If $X$ is a metric space and $x \in X$, let $\delta_{x}$ denote the probability measure on $X$ degenerate on $x$. Letting

$$
H_{i}=\left\{C_{i} \in P_{i}: \int_{P} \int_{T} q_{i} \mathrm{~d} \mu \mathrm{d}\left(\delta_{C_{i}} \times \alpha_{-i}\right)>\int_{P} \int_{T} q_{i} \mathrm{~d} \mu \mathrm{d}\left(\alpha_{i} \times \alpha_{-i}\right)\right\}
$$

it follows from Simon and Zame (1990, Step 4) that

$$
\alpha_{i}\left(H_{i}\right)=\alpha_{i}\left(\left\{x_{i} \in P_{i}: \int_{P} \hat{q}_{i} \mathrm{~d}\left(\delta_{C_{i}} \times \alpha_{-i}\right)>\int_{P} \hat{q}_{i} \mathrm{~d}\left(\alpha_{i} \times \alpha_{-i}\right)\right\}\right)=0 .
$$

Step 5: Perturbation. As in Step 5 of Simon and Zame (1990), for all $i$, let $p^{i}: T \times P \rightarrow \mathbb{R}^{m}$ be any measurable selection from $Q$ which minimizes the $i$ th component. Let $Y=\left\{C \in P: C_{i} \in H_{i}\right.$ for at least two indices $\left.i\right\}$ and define $f: T \times P \rightarrow \mathbb{R}^{m}$ as follows:

$$
f(t, C)= \begin{cases}p^{i}(t, C) & \text { if } C \in H_{i} \times P_{-i} \text { but } C \notin Y, \\ q(t, C) & \text { otherwise. }\end{cases}
$$

Since $\alpha\left(H_{i} \times P_{-i}\right)=0$ for all $i \in I$, then

$$
\int_{P} \int_{T} f \mathrm{~d} \mu \mathrm{d} \alpha=\int_{P \backslash \cup_{i=1}^{m}\left(H_{i} \times P_{-i}\right)} \int_{T} q \mathrm{~d} \mu \mathrm{d} \alpha=\int_{P} \int_{T} q \mathrm{~d} \mu \mathrm{d} \alpha .
$$


Therefore,

$$
\int_{P} \hat{f} \mathrm{~d} \alpha=\int_{P} \hat{q} \mathrm{~d} \alpha
$$

Let $i \in I$. If $C_{i} \notin H_{i}$, then

$$
\hat{q}_{i}(C)=\hat{f}_{i}(C)
$$

except possibly for $C \in\left[\left\{C_{i}\right\} \times P_{-i}\right] \cap\left[\cup_{j \neq i}\left(H_{j} \times P_{-j}\right)\right]$. Finally, we also have that

$$
\hat{p}_{i}^{i}(C)=\hat{f}_{i}(C)
$$

for all $C \in H_{i} \times P_{-i}$ and $C \notin Y$.

Step 6: Solution. Note that $p_{i}^{i}: T \times P \rightarrow \mathbb{R}$ is lower semi-continuous in $C$ for all $t \in T$, as in Simon and Zame (1990, step 6). Thus, it follows from Fatou's Lemma (see Aliprantis and Border (1999, Theorem 11.19, p. 407)) that $\hat{p}_{i}^{i}: P \rightarrow \mathbb{R}$ is lower semicontinuous. Because of equations (34), (35) and (36), it follows from Simon and Zame (1990, Step 6) that $\alpha$ is a Nash equilibrium of the normal-form game $\left(P_{1}, \ldots, P_{m}, \hat{f}\right)$. Since $\hat{f}=\int_{T} f \mathrm{~d} \mu$, it follows that $(f, \alpha)$ is a solution of $G=\left(P_{1}, \ldots, P_{m}, T, \mu, Q\right)$.

This completes the proof of Theorem 2 .

\section{A.2 Proof of Lemma 1}

For convenience, let $S=T \times P$. Note first that $\Lambda$ is measurable by Theorem 17.18 in Aliprantis and Border (1999, p. 570) and has nonempty, convex and compact values. Furthermore, by Berge's Maximum Theorem (see Berge (1997, p. 116)), the correspondence $C \mapsto \Lambda(t, C)$ is upper hemi-continuous for all $t \in T$.

Then, $Q$ is integrably bounded since $\pi$ is integrably bounded and $Q$ is nonempty valued since $\Lambda$ is also nonempty valued. Since for all $s \in S, \Lambda(s)$ is compact, $Q(s)=$ $h(s, \Lambda(s))$ and $\lambda \mapsto h(s, \lambda)$ is continuous, then $Q(s)$ is compact. Thus, $Q$ is compact valued.

Since $\Lambda$ is convex valued, then $Q$ is convex valued as well. Indeed, if $s \in S$, $x_{1}, x_{2} \in Q(s)$ and $a \in(0,1)$, then there exists $\lambda_{l} \in \Lambda(s)$ such that $x_{l}=h\left(s, \lambda_{l}\right)$ for all $l=1,2$. Then, $a \lambda_{1}+(1-a) \lambda_{2} \in \Lambda(s)$ and $a x_{1}+(1-a) x_{2}=h\left(s, a \lambda_{1}+(1-a) \lambda_{2}\right)$ imply that $a x_{1}+(1-a) x_{2} \in Q(s)$. 
Since $C \mapsto \Lambda(t, C)$ is upper hemi-continuous and $(C, \lambda) \mapsto h(t, C, \lambda)$ is continuous for all $t \in T$, then $C \mapsto Q(t, C)$ is upper hemi-continuous.

Finally, we show that $Q$ is measurable. Define $\Xi: S \rightrightarrows S \times \Delta(\mathbb{K})$ by $\Xi(s)=$ $\{(s, \lambda): \lambda \in \Lambda(s)\}$.

We claim that $\Xi$ is measurable. Since $\Xi$ is compact valued, then it is enough to show that $\Xi$ is weakly measurable (see Aliprantis and Border (1999, Lemma 17.2, p. 559)). Let $A$ and $B$ be measurable subsets of $S$ and $\Delta(\mathbb{K})$, respectively. Then, $\Xi^{\ell}(A \times B)=\{s \in S: \Xi(s) \cap(A \times B) \neq \emptyset\}=A \cap \Lambda^{\ell}(B)$ is measurable since $\Lambda$ is measurable. Therefore, if $V=\cup_{k=1}^{\infty}\left(A_{k} \times B_{k}\right)$ and $A_{k}$ and $B_{k}$ are measurable subsets of $S$ and $\Delta(\mathbb{K})$, respectively, for all $k \in \mathbb{N}$, then $\Xi^{\ell}(V)=\cup_{k=1}^{\infty} \Xi^{\ell}\left(A_{k} \times B_{k}\right)$ is measurable. Therefore, if $V$ is an open subset of $S \times \Delta(\mathbb{K})$, then there exist sequences $\left\{A_{k}\right\}$ and $\left\{B_{k}\right\}$ of open subsets of $S$ and $\Delta(\mathbb{K})$ such that $V=\cup_{k=1}^{\infty}\left(A_{k} \times B_{k}\right)$ since both $S$ and $\Delta(\mathbb{K})$ are second countable. Thus, $\Xi^{\ell}(V)$ is measurable and so $\Xi$ is weakly measurable.

Since $\Xi$ is measurable, then $Q$ is measurable as well. In fact, let $B$ be a measurable subset of $\mathbb{R}^{m}$. Then, $h^{-1}(B)$ is a measurable subset of $S \times \Delta(\mathbb{K})$ and so $Q^{\ell}(B)=$ $\left\{s \in S: \Xi(s) \cap h^{-1}(B) \neq \emptyset\right\}=\Xi^{\ell}\left(h^{-1}(B)\right)$ is measurable. This completes the proof of Lemma 1.

\section{A.3 Properties of $\varphi$}

In this appendix, we establish the properties of the agent's constraint correspondences $\varphi^{P M}, \varphi^{M S}$ and $\varphi^{H}$. In all these cases, the result is a consequence of the following Lemma.

Lemma 5 If $\phi: P \rightrightarrows \mathbb{K}$ is continuous with nonempty compact values, then $\varphi$ : $T \times P \rightrightarrows \Delta(\mathbb{K})$ defined by

$$
\varphi(t, C)=\{\lambda \in \Delta(\mathbb{K}): \lambda(\phi(C))=1\}
$$

is continuous and has nonempty, convex, compact values.

Proof. It follows from Aliprantis and Border (1999, Theorem 16.14, p. 530) that $\varphi$ is upper hemi-continuous with nonempty, compact, convex values. 
We claim that $\varphi$ is also lower hemi-continuous. In order to prove this claim, let $\left\{t_{n}\right\}_{n=1}^{\infty}$ be a convergent sequence in $T,\left\{C_{n}\right\}_{n=1}^{\infty}$ be a convergent sequence in $P, t=\lim _{n} t_{n}, C=\lim _{n} C_{n}$ and $\lambda \in \varphi(t, C)$. We need to prove that there exists a subsequence $\left\{n_{j}\right\}_{j=1}^{\infty}$ of indexes and elements $\lambda_{n_{j}} \in \varphi\left(t_{n_{j}}, C_{n_{j}}\right)$ such that $\lambda_{n_{j}}$ converges to $\lambda$.

By Aliprantis and Border (1999, Theorem 16.16, p. 531), the function $\Phi$ from $P$ into the space of all nonempty, compact subsets of $\mathbb{K}$ endowed with the Hausdorff metric defined by $\Phi(C)=\phi(C)$ is continuous. Thus, letting $d_{H}$ denote the Hausdorff metric, it follows that $d_{H}\left(\phi\left(C_{n}\right), \phi(C)\right)$ converges to zero.

For all $j \in \mathbb{N}$, let $n_{j} \in \mathbb{N}$ be such that $d_{H}\left(\phi\left(C_{n_{j}}\right), \phi(C)\right)<1 / j$. Let $j$ be fixed. Since $d_{H}\left(\phi\left(C_{n_{j}}\right), \phi(C)\right)<1 / j$, then $\phi(C) \subseteq \cup_{k \in \phi\left(C_{n_{j}}\right)} B_{1 / j}(k)$. Since $\phi(C)$ is a compact subset of $\mathbb{K}$, there exists $\left\{k_{1}, \ldots, k_{M}\right\} \subseteq \phi\left(C_{n_{j}}\right)$ such that $\phi(C) \subseteq \cup_{m=1}^{M} B_{1 / j}\left(k_{m}\right)$.

Finally, define $B_{1}=B_{1 / j}\left(k_{1}\right), B_{m}=B_{1 / j}\left(k_{m}\right) \backslash \cup_{l=1}^{m-1} B_{l}$ for all $m=2, \ldots, M$, and $\lambda_{n_{j}}$ by setting $\lambda_{n_{j}}\left(k_{m}\right)=\lambda\left(B_{m}\right)$ for all $m=1, \ldots, M$.

Since $\lambda(\phi(C))=1$, then $\lambda_{n_{j}}\left(\phi\left(C_{n_{j}}\right)\right)=1$ and, arguing as in the proof of Parthasarathy (1967, Theorem II.6.3), it follows that $\lambda_{n_{j}}$ converges to $\lambda$. Therefore, $\varphi$ is lower hemicontinuous.

As a consequence, we obtain the following corollary.

Corollary 2 The correspondences $\varphi^{P M}, \varphi^{M S}$ and $\varphi^{H}$ are continuous with nonempty, convex, compact values.

Proof. In the case of $\varphi^{M S}$, simply define $\phi^{M S}: P \rightrightarrows \mathbb{K}^{M S}$ by $\phi^{M S}(C)=C$. Clearly, $\phi^{M S}$ is continuous and has nonempty compact values.

In the case $\varphi^{P M}$, we define for all $i \in I, \phi_{i}: P \rightrightarrows \mathbb{K}^{P M}$ by $\phi_{i}(C)=\{i\} \times C_{i}$ and $\phi^{P M}: P \rightrightarrows \mathbb{K}^{P M}$ by $\phi^{P M}(C)=\cup_{i=1}^{m} \phi_{i}(C)$. Clearly, $\phi^{P M}$ has nonempty and compact values and $\phi_{i}$ is continuous for all $i \in I$. Since the finite union of continuous correspondences is continuous (see Aliprantis and Border (1999, Theorem 16.27, p. 537)), $\phi^{P M}$ is continuous.

In the hybrid case, we define for all $i \in I, \phi_{i}: P \rightrightarrows \mathbb{K}^{H}$ by, $\phi^{i}(C)=\{i\} \times C_{i}$, then, for each $n$ define $\phi_{n}(C)=\cup_{i \in I_{n}} \phi^{i}(C)$, where $\left\{I_{n}\right\}_{n=1}^{N}$ is a finite partition of $I$, it 
is easy to see that $\phi_{n}$ has nonempty and compact values and $\phi_{n}$ is continuous for all $n$. Finally, define $\phi^{H}: P \rightrightarrows \mathbb{K}^{H}$ by $\phi^{H}(C)=\prod_{n=1}^{N} \phi_{n}(C)$. Since the finite product of continuous correspondences with compact values is continuous (see Aliprantis and Border (1999, Theorem 16.28, p. 537)), $\phi^{H}$ is continuous and has nonempty and compact values as well.

In all cases the conclusion follows from Lemma 5.

\section{References}

Aliprantis, C., And K. Border (1999): Infinite Dimensional Analysis. Springer, Berlin.

Berge, C. (1997): Topological Spaces. Dover, New York.

Diestel, J., And J. J. Uhl (1977): Vector Measures. American Mathematical Society, Providence.

Furukawa, N. (1972): "Markovian Decision Processes with Compact Action Spaces," The Annals of Mathematical Statistics, 43(5), 1612-1622.

Hildenbrand, W. (1974): Core and Equilibria of a Large Economy. Princeton University Press, Princeton.

Martimort, D. (2006): "Multi-Contracting Mechanism Design," in Advances in Economics and Econometrics Theory and Applications, Ninth World Congress, Volume 1, ed. by R. Blundell, W. K. Newey, and T. Persson. Cambridge University Press, Cambridge.

Martimort, D., And A. Semenov (2006): "Ideological Uncertainty and Lobbying Competition," mimeo, IDEI Toulouse.

Martimort, D., and L. Stole (2002): "The Revelation and Delegation Principles in Common Agency Games," Econometrica, 70(4), 1659-1673. 
- (2003): "Contractual Externalities and Common Agency Equilibria," Advances in Theoretical Economics, 3, Article 4.

MÄrz, M., And R. M. ShorrT (1994): "Weak Convergence of Vector Measures," Publ. Math. Debrecen, 45, 71-92.

Monteiro, P., and F. Page (2008a): "Catalog Competition and Nash Equilibrium in Nonlinear Pricing Games," Economic Theory, 34, 503-524.

- (2008b): "Endogenous Mechanisms and Nash Equilibrium in Competitive Contracting Games," Journal of Mathematical Economics, doi:10.1016/j.jmateco.2008.05.003.

Page, F., And P. Monteiro (2003): "Three Principles of Competitive Nonlinear Pricing," Journal of Mathematical Economics, 39, 63-109.

Parthasarathy, K. (1967): Probability Measures on Metric Spaces. Academic Press, New York.

Peters, M. (2001): "Common Agency and the Revelation Principle," Econometrica, 69(5), 1349-1372.

_ (2003): "Negotiation and Take-It-Or-Leave-It in Common Agency," Journal of Economic Theory, 111(1), 88-109.

Simon, L., And W. Zame (1990): "Discontinuous Games and Endogenous Sharing Rules," Econometrica, 58(4), 861-872. 